\title{
A study on correlation between surface measurement of limbs and underlying tendon grafts used for reconstructive surgeries
}

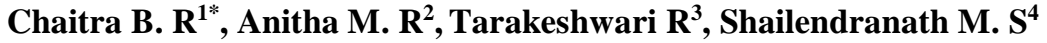 \\ ${ }^{1,3}$ Assistant Professor, ${ }^{2}$ Professor, ${ }^{4}$ Professor and Head, Dept. of Anatomy, Akash Institute of Medical Sciences and Research \\ Center, Devanahalli, Bengaluru Rural, Karnataka, India \\ *Corresponding Author: Chaitra B. R \\ Email: drchaitrabr@gmail.com
}

Received: $9^{\text {th }}$ August, 2018

Accepted: $13^{\text {th }}$ August, 2018

\begin{abstract}
Introduction: Tendon transfer restores balance to the hand which has lost its power due to tendon or muscle destruction. There are various factors which are taken into account while selecting a graft for reconstructive surgeries such as availability, length, expendability of graft tendon. The purpose of this study is to correlate the surface measurement of upper limb and lower limb using anatomical landmarks and underlying tendon lengths used for grafting. This will provide an idea for estimation of underlying donor tendon lengths by easily measured surface measurements of upper and lower limbs.

Materials and Methods: The study was conducted on 30 cadaveric upper and lower limbs collected in the department of anatomy, AIMS\&RC, Devanahalli, Bengaluru. Lengths of commonly harvested tendons used for grafting were measured. In upper limb, tendons of palmaris longus, extensor digiti minimi and extensor indices were selected. In lower limb, tendons of plantaris and extensor digitorum longus of $2^{\text {nd }}$ toe were selected. Surface measurement of upper limb and lower limbs were measured using bony land marks. Correlation between surface measurement of limbs and lengths of underlying tendons used for grafting were analyzed. By using, the univariate least square regression model, tendon multiplier is generated for each tendon, which gives the tendon length in donors by using the surface measurements of upper and lower limbs.

Results: Mean tendon lengths of palmaris longus is $15.53 \pm 4.7 \mathrm{~cm}$, extensor digiti minimi is $19.7 \pm 1.9 \mathrm{~cm}$, extensor indices is $19.1 \pm 1.2 \mathrm{~cm}$, plantaris is $35.97 \pm 2.7 \mathrm{~cm}$ and extensor digitorum longus of second toe is $27.93 \pm 2.9 \mathrm{~cm}$. Tendon multiplier for the tendon of palmaris longus is 0.66 , for extensor digiti minimi is 0.53 , for extensor indices is 0.31 , for plantaris is 0.91 and extensor digitorum longus is 0.51 . Mean surface measurement of upper limb is $27.5 \pm 2.31 \mathrm{~cm}$ and lower limb is $37.43 \pm 2.6 \mathrm{~cm}$.

Conclusion: While selecting tendons for graft, length of donor tendon is a limiting variable. Using the easily available surface measurements, surgeons may be able to better estimate the underlying tendon lengths available for grafting.
\end{abstract}

Keywords: Tendon, Plantaris, Palmaris longus, Graft, Reconstruction.

\section{Introduction}

Extensor tendons in the dorsum of hand and distal forearm are quite superficially located. So, any injuries in these areas will commonly cause underlying tendon injury. ${ }^{1}$ Tendons which are injured commonly require reconstruction with tendon grafts. ${ }^{2}$ There are certain criteria that the tendon graft should meet, such as, availability of graft, low donor site morbidity, sufficient length and diameter, for longer and multiple tendon reconstruction. ${ }^{3}$

Earlier it was thought that two stage tendon reconstruction gives better results than one stage procedures. In 2005, the IFSSH, flexor tendon committee has encouraged single stage grafting, as current suture techniques are stronger and rehabilitation has been given more importance than in the past. ${ }^{4}$ Most commonly used donor tendons for grafting are, tendons of palmaris longus (PL), extensor digiti minimi (EDM) and extensor indices (EI) in upper limb. In lower limb, Plantaris (Pt) and extensor digitorum longus of second toe (EDL) are used. ${ }^{5}$

Prior knowledge about the available tendon lengths would help surgeons in preoperative planning and selection of donors for grafting. It also aids the surgeons in counselling the patient before surgery, location of graft fixation and expected incisions. ${ }^{6}$ Objectives of this study is to find the a) length of tendons used for grafting and anatomical surface measurements of upper and lower limbs. b) a method to estimate the length of donor tendons used for grafting by using easily available anatomical surface measurements of limbs. c) correlation between tendon lengths and surface measurements of right and left sided upper and lower limbs.

\section{Materials and Methods}

The study was conducted in 30 cadaveric upper and lower limbs collected in the department of anatomy, AIMS \&RC, Devanahalli, Bengaluru rural. Before performing the dissection, anatomical surface measurements of upper and lower limbs were taken using a ruled tape measure. Upper limb was taken from tip of olecranon to ulnar styloid. Lower limb length was taken from head of fibula to tip of lateral malleolus.

Lengths of commonly harvested tendon grafts were measured using rule tape measure. In upper limb, tendons of palmaris longus, extensor digiti minimi and extensor indices were selected. In lower limb, tendons chosen were, plantaris and extensor digitorum longus of second toe. Lengths of tendons were measured from their 
proximal end, which we defined as the most distal point of the muscle- tendon junction to the distal end, that is bony insertion. For plantaris, distal end was taken as a point where a line connecting the pisiform and tubercle of scaphoid bone crosses the tendon of palmaris longus. For extensor digiti minimi and indices, distal point was taken as head of distal phalanx of little finger and index finger respectively. For plantaris, distal point was taken as posterior surface of calcaneus. For extensor digitorum longus, distal point was taken as head of distal phalanx of $2^{\text {nd }}$ toe.

Analysis was made to find the correlation between anatomical surface measurements of limbs and lengths of underlying tendons used for grafting. Linear model was generated which gives tendon lengths by using anatomical surface measurements of limbs. For each tendon, a tendon multiplier was generated by using a univariate ordinary least square regression model (OLS), that is used to predict the length of donor tendons used for grafting by using easily available anatomical surface measurement of limbs. According to the model, tendon length is generated by using the formula given below.

Tendon length $=\beta 0+\beta 1 \times$ surface measurement $+€$

Tendon length is the length of tendons measured in cadaveric upper and lower limbs. Surface measurement is the ulnar length for upper limb and fibular length for lower limb. $\beta 0$ and $\beta 1$ are the regression coefficients. $\beta 0$ is interpreted as constant and $\beta 1$ is interpreted as tendon multiplier. $€$ can be interpreted as the error not described in the model.

\section{Results}

Mean tendon lengths of palmaris longus, EDM, EI, plantaris and EDL along with the mean surface measurements of upper and lower limbs are given in Table 1. Forearm length correlated with underlying tendon length of palmaris longus $\left(\mathrm{R}^{2}=0.105\right), \mathrm{EDM}\left(\mathrm{R}^{2}\right.$ $=0.412)$, EI $\left(R^{2}=0.324\right)$. Fibular length correlated with underlying tendon lengths of plantaris $\left(\mathrm{R}^{2}=0.733\right)$ and EDL $\left(\mathrm{R}^{2}=0.214\right)$, where $\mathrm{R}^{2}$ is the coefficient of variation.

Fig. 2 and 3 shows the graphic representation of the relation between surface measurements of limbs and tendon lengths of palmaris longus and plantaris. Correlation is highest for tendons of plantaris, palmaris longus followed by EDM, EDL and EI. Tendon multiplier generated by linear regression model is significant with $\mathrm{p}$ value $<0.05$ for all tendons except for EI. (Table 2).

Mean tendon lengths of all tendons of right limbs are $1 \mathrm{~cm}$ more than that of left limb, except for palmaris longus and EDM (Table 3). For tendon of palmaris longus, mean tendon length of left limb is more than right limb by $3 \mathrm{~cm}$. Mean tendon length of EDM of right and left limb are almost the same.

Table 1: Summary of length of tendons and surface measurements of upper and lower limb in $\mathrm{cm}$

\begin{tabular}{|l|c|c|c|}
\hline \multirow{2}{*}{ Tendons } & \multirow{2}{*}{$\mathbf{N}$} & \multicolumn{2}{|c|}{ Length in cm } \\
\cline { 3 - 4 } & & Mean & SD \\
\hline Palmaris longus & 30 & 15.53 & 4.77 \\
\hline EDM & 30 & 19.7 & 1.91 \\
\hline EI & 30 & 19.1 & 1.26 \\
\hline Plantaris & 30 & 35.97 & 2.78 \\
\hline EDL & 30 & 27.93 & 2.9 \\
\hline Surface measurement & & & \\
\hline Upper limb & 30 & 27.5 & 2.3 \\
\hline Lower limb & 30 & 37.43 & 2.6 \\
\hline
\end{tabular}

Table 2: Estimated tendon multiplier for various tendons of upper and lower limb

\begin{tabular}{|l|c|c|c|c|}
\hline Tendon & $\boldsymbol{\beta ~ 0}$ & $\boldsymbol{\beta ~ 1}$ & $\mathbf{R}^{\mathbf{2}}$ & P value \\
\hline Palmaris longus & -2.85 & 0.66 & 0.1 & $<0.001^{*}$ \\
\hline EDM & 5.11 & 0.53 & 0.41 & $0.01^{*}$ \\
\hline EI & 10.52 & 0.31 & 0.32 & 0.08 \\
\hline Plantaris & 1.74 & 0.91 & 0.73 & $<0.001^{*}$ \\
\hline EDL & 8.61 & 0.51 & 0.21 & $<0.001^{*}$ \\
\hline
\end{tabular}

$\beta 0$ - constant

$\beta 1$-Tendon multiplier

$\mathrm{R}^{2}$ - Coefficient of variation

*Tendon multiplier $(\beta 1)$ generated was significant for all tendons (p value is $<0.05$ ) except for EI. 
Table 3: Comparison between tendon lengths and surface measurements of upper and lower limbs of right and left side

\begin{tabular}{|l|c|c|c|c|c|c|}
\hline \multirow{2}{*}{ Tendons } & \multicolumn{3}{|c|}{ Right limb } & \multicolumn{3}{c|}{ Left limb } \\
\cline { 2 - 7 } & $\mathbf{N}$ & Mean & SD & N & Mean & SD \\
\hline Palmaris longus & 19 & 14.26 & 5.41 & 11 & 17.73 & 2.24 \\
\hline EDM & 19 & 19.74 & 2.05 & 11 & 19.64 & 1.74 \\
\hline EI & 19 & 19.32 & 1.1 & 11 & 18.73 & 1.48 \\
\hline Plantaris & 16 & 36.25 & 2.64 & 14 & 35.64 & 3.1 \\
\hline EDL & 16 & 28.31 & 2.89 & 14 & 27.5 & 2.98 \\
\hline \multicolumn{7}{|c|}{ Surface Measurement } \\
\hline Upper limb & 19 & 27.68 & 2.45 & 11 & 27.18 & 2.13 \\
\hline Lower limb & 16 & 37.63 & 2.52 & 14 & 2.77 & 2.77 \\
\hline
\end{tabular}

\section{Fig. 1: Measurement of length of tendon of Palmaris longus}

1. Tendon of palmaris longus

2. Musculotendinous junction - Proximal point of tendon measurement

3. Line joining tubercle of scaphoid and pisiform - Distal point of tendon measurement

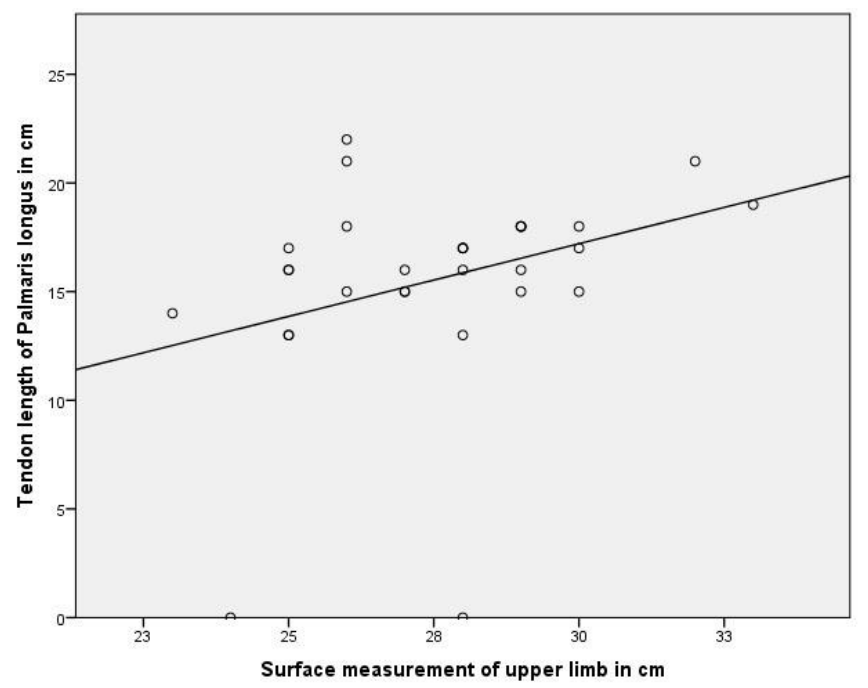

Fig. 2: Correlation between surface measurement of upper limb and tendon length of palmaris longus in $\mathrm{cm}$ 


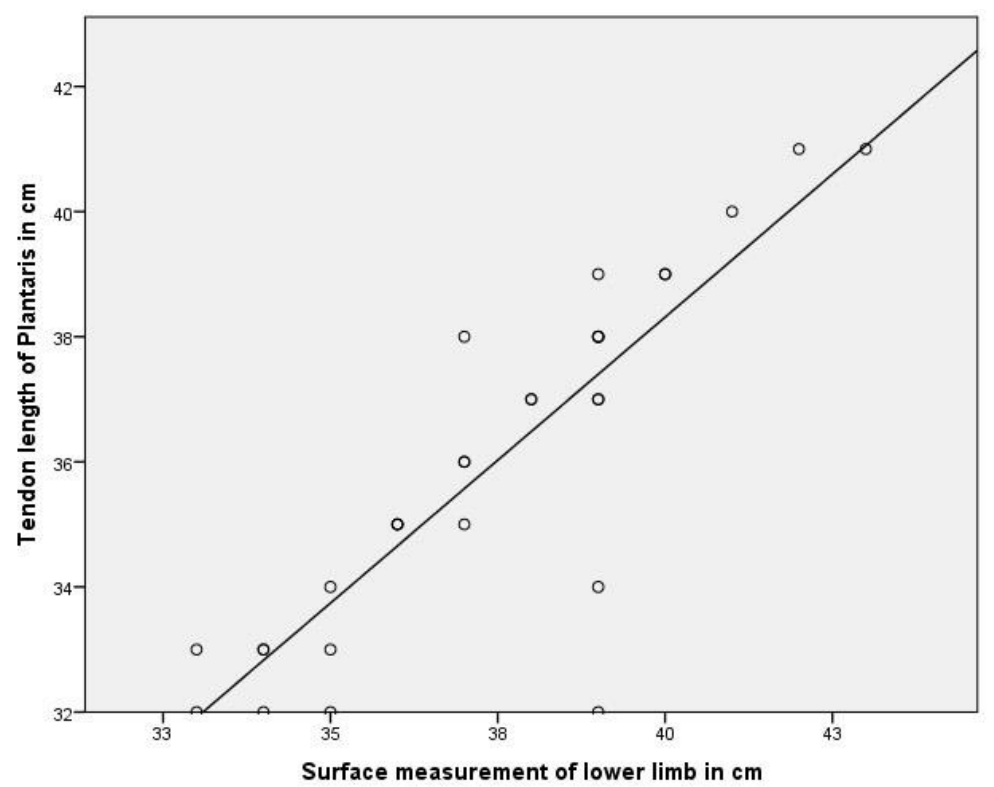

Fig. 3: Correlation between surface measurement of lower limb and tendon length of plantaris in $\mathrm{cm}$.

\section{Discussion}

Mean tendon lengths of all the tendons found in this study are comparable to those reported by Wehbe $\mathrm{MA}^{6}$ and Milone MT et al. ${ }^{7}$ Mean tendon length of palmaris longus is within $2 \mathrm{~cm}$, plantaris within $4 \mathrm{~cm}$, EDL within $3 \mathrm{~cm}$, in comparable to those reported by Wehbe MA. ${ }^{6}$
Mean tendon length of EDM and EI shows $9 \mathrm{~cm}$ different from that reported by Wehbe $\mathrm{MA}^{6}$ and Milone MT et al. ${ }^{7}$ (Table 4).

In the present study, while measuring the length of tendons of EDM and EI, distal limit was taken as distal phalanx as these tendons reach distal phalanx through extensor expansion.

Table 4: Comparison of mean tendon lengths of various tendons in $\mathrm{cm}$ with previous studies

\begin{tabular}{|l|c|c|c|}
\hline \multicolumn{1}{|c|}{ Tendons } & ${\text { Wehbe } \mathbf{M A}^{\mathbf{6}}}$ & Milone MT et al $^{\mathbf{7}}$ & Present study $^{\text {Maris longus }}$ \\
\hline Palmaris & 13.1 & 15.5 \\
\hline EDM & 11 & 10.8 & 19.7 \\
\hline EI & 10 & 10.6 & 19.1 \\
\hline Plantaris & 31 & 29.2 & 35.9 \\
\hline EDL & 30 & 28.4 & 27.9 \\
\hline
\end{tabular}

Tendon multiplier $(\beta 1)$ which is generated for each tendon of upper and lower limb by using univariate linear regression model was comparable to those reported by Milone $\mathrm{MA}^{7}$ (Table 5). It is possible to estimate the length of donor tendons used for grafting by using the easily measured surface measurement of upper and lower limbs, tendon multiplier $(\beta 1)$ and constant $(\beta 0)$ from the formula of univariate regression model.

Table 5: Comparison between tendon multiplier ( $\beta$ 1) generated for various tendons of upper and lower limb with other studies

\begin{tabular}{|l|c|c|}
\hline \multirow{2}{*}{ Tendons } & \multicolumn{2}{c|}{ Tendon multiplier ( $\beta$ 1) } \\
\cline { 2 - 3 } & Milone MA & Present study \\
\hline Palmaris longus & 0.51 & 0.66 \\
\hline EDM & 0.18 & 0.53 \\
\hline EI & 0.2 & 0.31 \\
\hline Plantaris & 0.69 & 0.91 \\
\hline EDL & 0.6 & 0.51 \\
\hline
\end{tabular}

Study shows that the length of tendons varies with variation in the length of extremity in the patients. Correlation was strongest for the tendons of plantaris and palmaris longus (Fig. 1 and 2). This study is an attempt to add further data to the existing literature on how the length of underlying tendons varies with anatomical surface measurement of limbs. This will help the surgeons to select the appropriate donor for grafting. 


\section{Conclusion}

Availability and adequate length of tendon are the two important criteria to be considered before selecting the tendons for grafting during reconstructive surgeries. If tendon is not long enough, more suitable tendon which has adequate length to be considered for grafting. It is very important to estimate the length of tendon graft before harvest. Correlation between surface measurements of limbs and lengths of tendons used for graft, gives an approximate estimate of tendon length used for harvest before selection for the graft.

\section{List of Abbreviations}

PL - Palmaris longus

EDM- Extensor digiti minimi

EI - Extensor indices

$\mathrm{Pt}$ - Plantaris

EDL - Extensor digitorum longus

$\mathrm{N}$ - Number of specimens

$\mathrm{SD}-$ Standard deviation

\section{References}

1. Amirthrajah M, Lattanza L. "Open extensor tendon injuries.” J Hand Surg Am. 2015;40:391-397.

2. Jakubietz MG, Jakubietz DF, Gruenert JG, Zahn R, Meffert RH, Jakubietz RG. "Adequacy of palmaris longus and plantaris tendons for grafting." JHS. 2016;36A:695-698.
3. Harvey FJ, Chu G, Harvey PM. "Surgical availability of plantaris tendon." J Hand Surg. 1983;8:243-247.

4. Amadio P, An KH, Ejeskar A, Guimbertau JC, Harris S, Savage R et al. "IFSSH flexor tendon committee report." $J$ Hand Surg. 2005;30B:100-116.

5. Moore T, Anderson B, Seiler III JG. "Flexor tendon reconstruction." JHS. 2010;35A:1025-1030.

6. Wehbe MA. "Tendon graft donor sites." J Hand Surg Am. 1992;17(6):1130-1132.

7. Milone MT, Strarecki M, Ayalon O, Arersano MW, Sapienza A. "The relationship between surface measurement and underlying tendon autograft length for upper extremity reconstructive surgery." J Hand Surg Am. 2017;42:663.e1664.e5.

How to cite this article: Chaitra B. R, Anitha M. R, Tarakeshwari R, Shailendranath M. S. A study on correlation between surface measurement of limbs and underlying tendon grafts used for reconstructive surgeries. Indian $\mathrm{J}$ Clin Anat Physiol. 2018;5(4):449-453. 